\title{
GIFTS SM EDU Data Processing and Algorithms
}

\author{
Jialin Tian*, David G. Johnson ${ }^{\dagger}$ Robert A. Reisse ${ }^{\ddagger}$, and Michael J. Gazarik ${ }^{\dagger}$ \\ *SSAI, MS 468, NASA Langley Research Center, Hampton, VA 23681-2199 \\ Email: Jialin.Tian-1@nasa.gov \\ $\dagger$ MS 434, NASA Langley Research Center, Hampton, VA 23681-2199 \\ $\ddagger$ MS 468, NASA Langley Research Center, Hampton, VA 23681-2199
}

\begin{abstract}
The Geosynchronous Imaging Fourier Transform Spectrometer (GIFTS) Sensor Module (SM) Engineering Demonstration Unit (EDU) is a high resolution spectral imager designed to measure infrared (IR) radiances using a Fourier transform spectrometer (FTS). The GIFTS instrument employs three Focal Plane Arrays (FPAs), which gather measurements across the long-wave IR (LWIR), short/mid-wave IR (SMWIR), and visible spectral bands. The raw interferogram measurements are radiometrically and spectrally calibrated to produce radiance spectra, which are further processed to obtain atmospheric profiles via retrieval algorithms. This paper describes the processing algorithms involved in the calibration stage. The calibration procedures can be subdivided into three stages. In the pre-calibration stage, a phase correction algorithm is applied to the decimated and filtered complex interferogram. The resulting imaginary part of the spectrum contains only the noise component of the uncorrected spectrum. Additional random noise reduction can be accomplished by applying a spectral smoothing routine to the phase-corrected blackbody reference spectra. In the radiometric calibration stage, we first compute the spectral responsivity based on the previous results, from which, the calibrated ambient blackbody (ABB), hot blackbody (HBB), and scene spectra can be obtained. During the post-processing stage, we estimate the noise equivalent spectral radiance (NESR) from the calibrated ABB and HBB spectra. We then implement a correction scheme that compensates for the effect of fore-optics offsets. Finally, for off-axis pixels, the FPA off-axis effects correction is performed. To estimate the performance of the entire FPA, we developed an efficient method of generating pixel performance assessments. In addition, a random pixel selection scheme is designed based on the pixel performance evaluation.
\end{abstract}

\section{INTRODUCTION}

The Geosynchronous Imaging Fourier Transform Spectrometer (GIFTS) Sensor Module (SM) Engineering Demonstration Unit (EDU) is a high resolution spectral imager designed to measure infrared (IR) radiances using a Fourier transform spectrometer (FTS). The GIFTS instrument employs three Focal Plane Arrays (FPAs), which gather measurements across the long-wave (685-1130 $\mathrm{cm}^{-1}$ ) IR (LWIR), short/mid-wave (1650-2250 $\mathrm{cm}^{-1}$ ) IR (SMWIR), and visible spectral bands. The raw interferogram measurements are radiometrically and spectrally calibrated to produce radiance spectra, which are further processed to obtain atmospheric profiles via retrieval algorithms. In this paper, we emphasize on the algorithmic components of the calibration process, which are divided in multiple stages. Prior to the radiometric calibration, the decimated and filtered complex interferograms must be phasecorrected so that their imaginary spectra contain only noise. The phase correction is performed on all three types of measurements for a series of scans, i.e., the ambient blackbody (ABB) reference, hot blackbody (HBB) reference, and scene scans. To reduce the random noise embedded in the reference measurements, a spectral smoothing method can be applied to the phase-corrected blackbody reference spectra. In the next stage, the calibrated $\mathrm{ABB}, \mathrm{HBB}$, and scene radiance spectra are computed based on the previous results. An assessment of the noise equivalent spectral radiance (NESR) is derived from the calibrated $\mathrm{ABB}$ and $\mathrm{HBB}$ references. During the post-processing stage, we implement two additional correction algorithms to compensate for the fore-optics offsets and FPA off-axis effects. In the last section, we introduce an efficient method of estimating the noise performance of the entire FPA, as well as a random pixel selection strategy that can be employed for generating a representative pixel sample set.

\section{Single-Pixel Calibration Algorithms}

For a single pixel element on the FPA, the calibration procedures involve the following steps: phase correction, spectral smoothing, responsivity calculation, radiometric calibration, NESR assessments, fore-optics offsets adjustment, and off-axis effects correction.

\section{A. Pre-calibration Algorithms}

1) Phase Correction: The phase correction algorithm is applied to the decimated and filtered complex interferogram $I_{m}(n)$. Its complex spectrum $N_{m}(\sigma)$ has the form of

$$
N_{m}(\sigma)=N(\sigma) e^{j \phi(\sigma)}+n(\sigma),
$$

where $n(\sigma)$ represents the noise vector, and $N(\sigma)$ is the real and noise-free spectrum. To estimate the phase function $\phi(\sigma)$, we adopt the Forman-Vanesse-Steel (FVS) method [1]. In this technique, a Hamming window $w(n)$, of length $W$, is applied to the complex interferogram with respect to the zero path difference (ZPD), i.e., $n=n_{z p d}$. The windowed interferogram is written as $I_{w}(n)=w(n) I_{m}(n)$, where

$$
w(n)= \begin{cases}0.54-0.46 \cos \left(\frac{2 \pi n}{W-1}\right), & \left|n-n_{z p d}\right| \leq \frac{W-1}{2} \\ 0, & \text { otherwise }\end{cases}
$$

The resulting interferogram $I_{w}(n)$ is circular shifted to obtain $I_{w}^{\prime}(n)$ such that $I_{w}^{\prime}(0)=I_{w}\left(n_{z p d}\right)$. The spectral response of $I_{w}^{\prime}(n)$ is computed via the Fourier transform, i.e., $N_{w}^{\prime}(\sigma)=$ $\mathcal{F}\left\{I_{w}^{\prime}(n)\right\}$, from which, the phase function can be estimated 


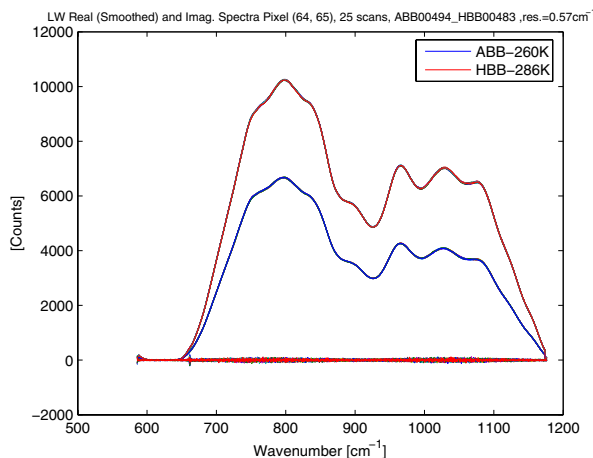

Fig. 1. Longwave phase-corrected and smoothed real and imaginary blackbody spectra at ambient $(260 \mathrm{~K})$ and hot (286K) temperatures, Pixel (64, 65), 25 scans.

as $\hat{\phi}(\sigma)=\tan ^{-1} \frac{\operatorname{Im}\left(N_{w}^{\prime}(\sigma)\right)}{\operatorname{Re}\left(N_{w}^{\prime}(\sigma)\right)}$. By applying the phase estimate, the phase-corrected spectrum becomes

$$
\widehat{N}(\sigma)=N(\sigma) e^{j(\phi(\sigma)-\hat{\phi}(\sigma))}+n(\sigma) e^{-j \hat{\phi}(\sigma)},
$$

and its resulting real and imaginary components are given as

$$
\begin{aligned}
& \operatorname{Re}\{\widehat{N}(\sigma)\} \approx N(\sigma)+\operatorname{Re}\{n(\sigma)\} \cos (\hat{\phi}(\sigma)), \\
& \operatorname{Im}\{\widehat{N}(\sigma)\} \approx \operatorname{Im}\{n(\sigma)\} \sin (\hat{\phi}(\sigma)) .
\end{aligned}
$$

Note that the imaginary part of the spectrum contains only noise, which can be discarded.

2) Spectral Smoothing: The phase correction is performed on three types of spectra, i.e., ABB, HBB, and scene. An optional smoothing algorithm can be applied to the blackbody references to improve the noise performance of the calibrated radiances. Two smoothing techniques are being considered for this purpose: the least-squares smoothing filter and the moving average (MA) filter. The MA smoothing method approximates the function within the predefined window as a constant whereas the least-squares algorithm fits a polynomial to all points within the window. The MA method does not preserve higher order moments, however, it is less demanding computationally. See [2], [3] for detailed reviews of these two techniques. When the final noise performance of these two methods are compared, no significant differences were observed; therefore, the MA smoothing technique is chosen due to its simplicity. Fig. 1 illustrates the phase corrected and smoothed longwave spectra for the real and imaginary blackbody references at ambient and hot temperatures.

\section{B. Radiometric Calibration}

To obtain the calibrated radiances, the first step involves the calculation of the spectral responsivity, which can be computed from

$$
R(\sigma)=\frac{\bar{N}^{H}(\sigma)-\bar{N}^{A}(\sigma)}{B^{H}(\sigma)-B^{A}(\sigma)} .
$$

$\bar{N}^{H}(\sigma)=\frac{1}{S} \sum_{i=1}^{S} N_{i}^{H}(\sigma)$ and $\bar{N}^{A}(\sigma)=\frac{1}{S} \sum_{i=1}^{S} N_{i}^{A}(\sigma)$ represent the mean $\mathrm{HBB}$ and $\mathrm{ABB}$ spectra averaged over $S$ scans, respectively. Using the relation of

$$
\bar{N}^{H}(\sigma)=R(\sigma)\left(B^{H}(\sigma)+O(\sigma)\right),
$$

the offset term can be solved from

$$
O(\sigma)=\frac{\bar{N}^{A}(\sigma) B^{H}(\sigma)-\bar{N}^{H}(\sigma) B^{A}(\sigma)}{\bar{N}^{H}(\sigma)-\bar{N}^{A}(\sigma)} .
$$

Next, the calibrated $\mathrm{HBB}, \mathrm{ABB}$, and scene radiance spectra can be determined, based on the responsivity and offset results, from

$$
\begin{aligned}
\widehat{B}_{i}^{H, A}(\sigma) & =\frac{N_{i}^{H, A}(\sigma)}{R(\sigma)}-O(\sigma), \\
\widehat{N}_{i}^{C}(\sigma) & =\frac{N_{i}^{C}(\sigma)}{R(\sigma)}-O(\sigma) .
\end{aligned}
$$

respectively. The variable $i$ denotes the scan number, i.e., $i=$ $1,2, \ldots, S$.

\section{Post-processing}

1) Noise Equivalent Spectral Radiance (NESR): This section describes the estimation of the noise equivalent spectral radiance (NESR) from calibrated blackbody references. The NESR is generally considered as a measure of the instrument noise performance, and can be estimated as the standard deviation of calibrated blackbody radiance spectra from multiple scans. The NESR definition is given by

$$
\operatorname{NESR}^{H, A}(\sigma)=\sqrt{\frac{1}{S} \sum_{i=1}^{S}\left(\widehat{B}_{i}^{H, A}(\sigma)-\overline{\widehat{B}^{H, A}}(\sigma)\right)^{2}} .
$$

Fig. 2 depicts the smoothed HBB and ABB NESR estimates over their corresponding non-smoothed NESRs computed from 25 interferogram scans.

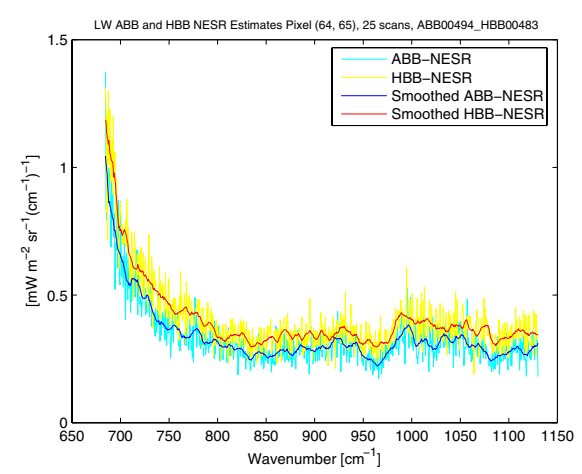

Fig. 2. Smoothed and non-smoothed longwave HBB and ABB NESR estimates computed from 25 interferogram scans.

2) Fore-optics Offsets: The previous calibration results are computed using internal blackbody references. To compensate for the offsets generated from fore-optics, a correction scheme is incorporated into the previous model. This can be achieved by using additional data measurements collected from an extended source. Given $\bar{C}_{e}^{H}(\sigma)$ and $\bar{C}_{e}^{A}(\sigma)$ as calibrated extended blackbody sources averaged over all scans, we have

$$
\bar{C}_{e}^{H, A}(\sigma)=R_{e}(\sigma) B_{e}^{H, A}(\sigma)+O_{e}(\sigma) .
$$


By solving for $R_{e}(\sigma)$ and $O_{e}(\sigma)$, the actual scene radiances can be approximated as

$$
\widetilde{N}_{i}^{C}(\sigma)=\frac{\widehat{N}_{i}^{C}(\sigma)-O_{e}(\sigma)}{R_{e}(\sigma)} .
$$

Fig. 3 shows the calibrated longwave $\mathrm{HBB}, \mathrm{ABB}$, and scene radiance spectra after performing the fore-optics offsets correction.

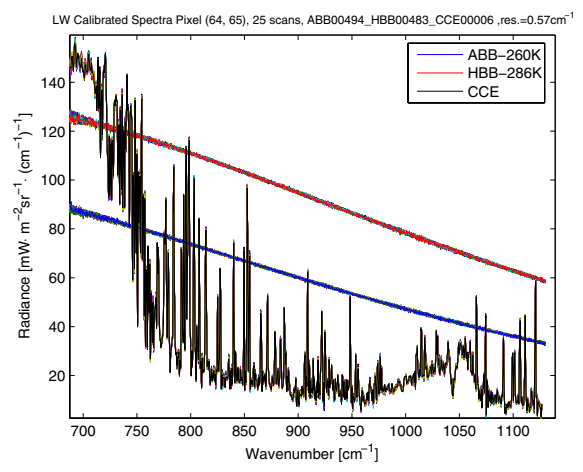

Fig. 3. Calibrated longwave $\mathrm{HBB}, \mathrm{ABB}$, and scene radiance spectra after the fore-optics offsets correction (25 scans).

3) Off-axis Effects: In this section, the correction of the FPA off-axis effects is presented. Since the GIFTS FPA contains $128 \times 128$ detector elements, the distortion caused by the off-axis effects is too great to be neglected. The off-axis pixels are sampled at slightly shorter optical path differences (OPDs), which cause the spectra of these pixels to expand to slightly higher wavenumbers [4]. The correction can be formulated as a fractional sampling rate conversion problem, which can be solved via sinc interpolations, i.e.,

$$
N_{i}^{C}(\sigma)=\sum_{m=0}^{M-1} \widetilde{N}_{i}^{C}\left(\sigma^{\prime}\right) \operatorname{sinc}\left(\sigma-\sigma^{\prime}\right), \quad \sigma^{\prime}=\frac{\sigma}{f},
$$

where $\sigma$ is the on-axis wavenumber scale, $\sigma^{\prime}$ is the off-axis scale, and $f$ is the off-axis factor that is computed from the FPA geometry. However, the sinc interpolation calculation is computational intensive, furthermore, the truncation window associated with the sinc kernels may cause overshoots in the resulting data. It has been shown that the zero-padding interpolation in the interferogram domain is equivalent to the sinc interpolation in the spectral domain except that the zero-padding method cannot evaluate the interpolation output at an arbitrary point [5]. To overcome this limitation, we designed the method of "over-padding", in which, we assign an over-padding factor $g$ that is closely correlated to the final resolution of the corrected spectra. For instance, if $f=0.9977$, and the length of the spectrum is $N$, then the zero-padded interferogram length is the roundoff value of $g * N / f$. If $g=100$, the actual off-axis factor of 0.997702 is obtained; if $g=1$, then the actual value of $f$ becomes 0.99758 due to roundoff errors. The corrected spectrum can be obtained by downsampling the Fourier transform of the over-padded interferogram by the factor $g$. Fig. 4 depicts the corrected spectra for two pixels, one pixel is located near the center of the FPA whereas the other is situated near the corner of the FPA. A better wavenumber alignment was achieved after performing the off-axis effects correction.

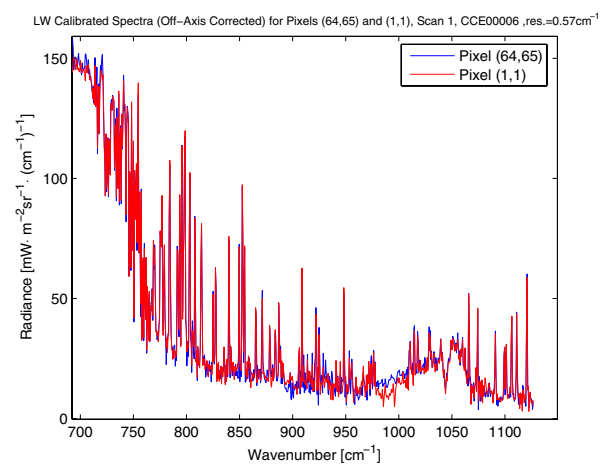

Fig. 4. Calibrated spectra for Pixel $(1,1)$ and Pixel $(64,65)$ after performing the off-axis effects correction.

\section{Focal Plane Array (FPA) Performance ASSESSMENT}

We have discussed the algorithmic procedures required for a single pixel measurement. To evaluate the entire FPA without completing all of the calculation steps, we designed a pixel inventory method, which estimates the FPA responsivity and noise distributions.

\section{A. Pixel Inventory}

The responsivity for any detector element can be approximated from the normalized intensity value at the ZPD, which is given by

$$
\widehat{R}^{x, y}=\frac{I_{m}^{x, y}\left(n_{z p d}\right)}{\frac{1}{X Y} \sum_{x} \sum_{y} I_{m}^{x, y}\left(n_{z p d}\right)} \forall x, y,
$$

where $x, y$ are pixel numbers. The FPA responsivity estimate is shown in Fig. 5(a). The FPA responsivity distribution within a predefined range of $0.8 \leq \widehat{R}^{x, y} \leq 1.2$ is plotted in Fig. 5(b). To assess the FPA noise, each interferogram is first normalized by its value at $n=n_{z p d}$, i.e.,

$$
\widetilde{I}_{m}^{x, y}(n)=\frac{I_{m}^{x, y}(n)}{I_{m}^{x, y}\left(n_{z p d}\right)} \forall x, y .
$$

Next, the estimated noise is computed from the root-meansquare value of the last $L$ samples in each interferogram that is represented by

$$
\widehat{N}^{x, y}=\sqrt{\frac{1}{L} \sum_{n=M-L}^{M-1}\left|\widetilde{I}_{m}^{x, y}(n)\right|^{2}} \forall x, y .
$$

Similarly, the FPA noise and its distribution are shown in Figs. 6(a) and 6(b), respectively. 


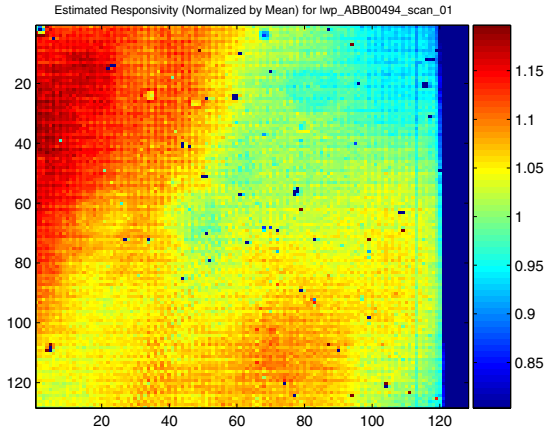

(a)



(b)

Fig. 5. Longwave pixel inventory: (a) FPA responsivity estimates, and (b) FPA responsivity distribution plot.

\section{B. Random Pixel Set Generation}

Once we have the knowledge of the FPA responsivity and noise estimates, a set of selection criteria is established by defining an acceptable range for the responsivity and noise. Thus, a random sample set of pixels can be chosen such that it reflects the statistical properties of the entire FPA. We can then perform the actual calibration procedures on this sample set. Fig. 7 shows a sample longwave pixel selection scheme, in which 64 random pixels are selected with 4 pixels per tap. The selection is uniformly distributed and unique within each tap.

\section{CONCLUSION}

This paper describes the required stages for calibrating the GIFTS SM EDU data spectrally and radiometrically. The following procedures were introduced: phase correction, spectral smoothing, radiometric calibration, NESR computation, correction algorithms for the fore-optics offsets and the offaxis effects. In the last section, methods for estimating the FPA's performance and generating random pixel sample sets were discussed.

\section{REFERENCES}

[1] M. L. Forman, W. H. Steel, and G. A. Vanasse, "Correction of asymmetric interferograms obtained in Fourier transform spectroscopy," J. Opt. Soc. Amer., vol. 56, pp. 59-63, 1966.

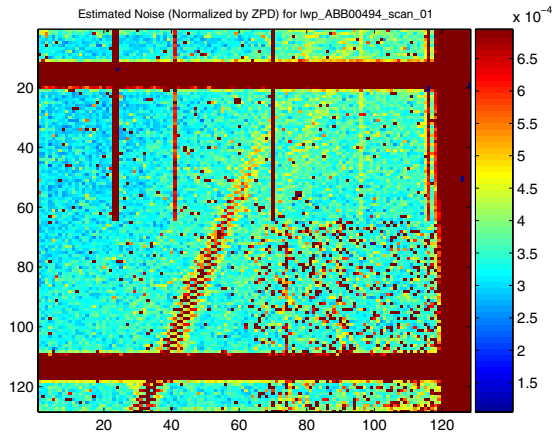

(a)

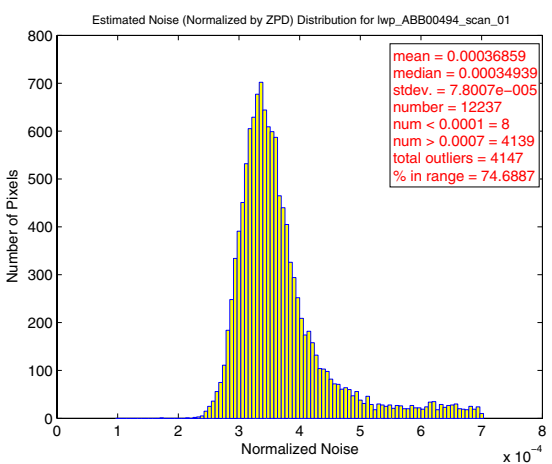

(b)

Fig. 6. Longwave pixel inventory: (a) FPA noise estimates, and (b) FPA noise distribution plot.

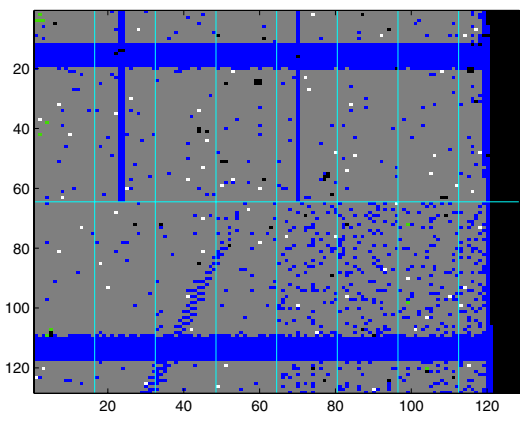

Fig. 7. A sample longwave random pixel set. Blue pixels: out-of-range noise; green pixels: out-of-range responsivity; black pixels: out-of-range noise and responsivity; gray pixels: satisfy both noise and responsivity constraints; and white pixels: random pixel selection.

[2] W. H. Press et al., Numerical Recipes in C: The Art of Scientific Computing, 2nd ed. Cambridge: Cambridge University Press, 1992.

[3] A. V. Oppenheim, R. W. Schafer, and J. R. Buck, Discrete-Time Signal Processing, 2nd ed. Upper Saddle River, NJ: Prentice Hall, 1999.

[4] J. Kauppinen and J. Partanen, Fourier Transforms in Spectroscopy. Berlin, Germany: WILEY-VCH, 2001.

[5] P. J. La Rivière and X. Pan, "Mathematical equivalence of zero-padding interpolation and circular sampling theorem interpolation with implication for direct Fourier image reconstruction," SPIE Conference on Image Processing, vol. 3338, pp. 1117-1126, 1998. 